\title{
Valvuloplastía mitral percutánea: experiencia del Hospital San Juan de Dios
}

\author{
*Humberto Dighero, *Flavio Zepeda, *Marcelo Soto, *Diego Godoy, *Ángel Puentes, \\ **Waldo Aranda, *Gorky Mori, *Pablo Sepúlveda, ${ }^{\star * \star B}$ Bruno Dighero. \\ * Laboratorio de Hemodinamia, Hospital San Juan de Dios. \\ ** Escuela de Salud Pública, Universidad de Chile. \\ *** Facultad de Medicina, Universidad de Chile.
}

\section{Resumen:}

Objetivo: Evaluar los resultados alejados de la valvuloplastia mitral percutánea (VMP), y analizar los factores asociados con eventos y reestenosis tardía.

Métodos y resultados: De 252 VPM realizadas entre diciembre de 1987 y diciembre de 2006, 245 pacientes tuvieron un mínimo de 6 meses de seguimiento y evaluación ecocardiográfica antes y después del procedimiento (97\%). La edad media fue de 46,3 $\pm 11,37$ años, las mujeres fueron $83,6 \%$. La media de seguimiento fue de $35,25 \pm 28,84$ meses, rango 6-132. La muerte cardiovascular, y el reemplazo de la válvula mitral o una segunda VMP fueron considerados como marcadores de peor resultado. El área de la válvula mitral aumentó de $1,0 \pm 0,17 \mathrm{~cm} 2$ a $1,71 \pm 0,31 \mathrm{~cm} 2$ ( $\mathrm{p}<0,0001)$ después del procedimiento. Durante el seguimiento, 53 pacientes $(21,6 \%)$ presentaron reestenosis, que fue predicha por un Score de Wilkins $>8(\mathrm{p}=$ $0,03)$. Cinco pacientes fallecieron $(2,04 \%), 22(8,9 \%)$ requirieron reemplazo de la válvula mitral y $11(4,5 \%)$ una segunda VMP. No encontramos variables clínicas o ecocardiográficas capaces de predecir estos eventos. Conclusiones: La VMP tuvo una tasa aceptable de complicaciones atribuibles al procedimiento. Las características ecocardiográficas de la válvula mitral se correlacionaron con la reestenosis. La incidencia de eventos tardíos es baja.

\section{Percutaneous mitral valvuloplasty: the san juan de dios hospital experience.}

Objective: To evaluate the long-term results of percutaneous mitral valvuloplasty (PMV), and analyze the factors associated with restenosis and late events.

Methods and results: Of 252 PMV performed between December 1987 and December 2006, 245 patients with a minimum of 6 months follow-up and echocardiographic evaluation before and after the procedure, were selected for long-term follow-up (97\%). The mean age was $46.3 \pm 11.37$ years old, $83.6 \%$ women. The mean follow-up was $35.25 \pm 28.84$ months, range 6-132. Cardiovascular death, and mitral valve replacement or second PMV, were considered as markers of worst outcome. The mitral valve area increased from $1.0 \pm 0.17 \mathrm{~cm} 2$ to $1.71 \pm 0.31 \mathrm{~cm} 2(\mathrm{p}<0.0001)$ after the first procedure. During follow up, 53 patients $(21.6 \%)$ developed restenosis, which was predicted by a Wilkins score $>8$ (p $=0.03)$. Five $(2.04 \%)$ patients died. Twenty-two $(8.9$ $\%)$ required mitral valve replacement and $11(4.5 \%)$ a second PMV. We found that no clinical or echocardiographic variables were able to predict these events.

Conclusions: PMV had an acceptable rate of complications attributable to the procedure. The echocardiographic characteristics of the mitral valve correlated with restenosis. The incidence of late events is low.

Key words: Mitral, valvuloplasty, percutaneous, follow-up.

\section{Correspondencia:}

Dr. Pablo Sepúlveda Varela

Laboratorio de Hemodinamia.

Departamento de Cardiología.

Hospital San Juan de Dios.

pablosep@gmail.com 


\section{Introducción:}

La estenosis mitral reumática (EM) es una enfermedad que afecta principalmente a mujeres jóvenes y que aún prevalece en Chile, aunque menos común que en el pasado ${ }^{1}$. Si su curso no se altera con el tratamiento adecuado, lleva a una alta morbilidad y mortalidad ${ }^{2-4}$. La valvuloplastía mitral percutánea (VMP) es una alternativa al tratamiento quirúrgico de la EM. Su papel en el cuidado de los pacientes con esta enfermedad ha seguido evolucionando en los últimos 25 años $^{5-7}$. En Chile, la primera VPM se llevó a cabo en nuestra institución en diciembre de 1987, por el Dr. Igor Palacios ${ }^{8}$. Los resultados que se obtienen con este procedimiento dependen de las características ecocardiográficas de la válvula mitral ${ }^{9-15}$. Los estudios de seguimiento, en el corto y mediano plazo han demostrado que los mejores resultados y más baja tasa de eventos que conducen a mejorar la supervivencia, se obtienen cuando el compromiso de la válvula mitral estimada por ecocardiografía, es menos pronunciada ${ }^{16-21}$. Sin embargo, a largo plazo los estudios de seguimiento son todavía escasos.

El objetivo de este estudio fue evaluar los resultados tardíos de la VMP y analizar los factores clínicos y ecocardiográficos que podrían estar relacionados con la reestenosis y otros eventos clínicos (muerte, segunda VMP, reemplazo valvular).

\section{Material y método:}

Pacientes: Entre Diciembre de 1987 y Diciembre de 2006, 252 pacientes portadores de EM sintomática y área valvular mitral $<1,5 \mathrm{~cm}^{2}$ fueron sometidos a VMP. Fueron excluidos del procedimiento los pacientes con más de 2 grados de regurgitación mitral según la clasificación Sellers ${ }^{12}$, o trombo en la aurícula izquierda, y / o anatomía muy desfavorable. Este grupo fue refinado a 245 pacientes con un mínimo de 6 meses de seguimiento y evaluación ecocardiográfica antes y después del procedimiento (97\% de seguimiento). La muerte, el reemplazo de la válvula mitral o una segunda VMP se considera como criterio de valoración de los peores resultados.

Procedimiento: La VMP fue efectuada por el método anterógrado transvenoso. Se utilizó la técnica del balón único descrito por Inoue y $\operatorname{cols}^{13}$, y la técnica de 2 balones descrita por $\mathrm{Al}$ Zeibag y cols ${ }^{14}$. El gasto cardíaco se midió por termodilución, y el área de la válvula se calculó mediante la fórmula de Gorlin ${ }^{9}$, antes y después de la dilatación.
Ecocardiografía: Se realizó antes de la dilatación, a las 24 horas, a los 30 días, a los 6 y 12 meses, y luego anualmente. El área de la válvula mitral se determinó mediante planimetría y el tiempo medio de presión como describió Hatle ${ }^{10}$. El puntaje ecocardiográfico se determinó por el método descrito por Wilkins y cols ${ }^{11}$, que considera la motilidad, engrosamiento y calcificación de la válvula y el aparato subvalvular, dando cada uno una puntuación de 1 a 4 . El grado de insuficiencia mitral se evaluó por el método de Sellers ${ }^{12}$. El resultado de la VMP se consideró óptima si el área de la válvula mitral fue $\geq 1,5 \mathrm{~cm} 2$ al final del procedimiento. Si el área que se obtuvo fue $<1,5 \mathrm{~cm}^{2}$, o si el paciente presentó insuficiencia mitral $>3$, el resultado se consideró sub-óptimo. La reestenosis fue definida como una pérdida de más del 50\% del incremento inicial del área obtenida tras la dilatación, o un área de $<1,5 \mathrm{~cm}^{2}$. Método estadístico: Para el análisis estadístico, se empleó la regresión logística multivariada en relación a la tasa de dilatación de la válvula mitral, la edad, la presencia de fibrilación auricular y la puntuación de Wilkins.

\section{Resultados:}

La edad promedio de la población de estudio fue de de 46,3 $\pm 11,37$ años de edad, siendo un $83,6 \%$ del género femenino. El seguimiento promedio de 35,25 \pm 28,84 meses (rango 6-132 meses) por evaluación en consulta externa o una entrevista telefónica. La distribución porcentual de las puntuaciones ecocardiográficas (puntuación de Wilkins) se muestra en la figura 1. Un resultado óptimo se obtuvo, según lo definido arriba, en el $82,9 \%$ de los pacientes y sub-óptimo en el $17,1 \%$ restante. El área inicial de la válvula mitral aumentó significativamente de $1,0 \pm 0,17 \mathrm{~cm} 2$ a 1,71 $\pm 0,31 \mathrm{~cm} 2(\mathrm{p}<0,0001)$ después de la PMV (Figura 2). Hubo una muerte atribuible al procedimiento, debido

Figura 1. Distribuci $\mathbf{n}$ seg $\mathbf{n}$ el Score de Wilkins.

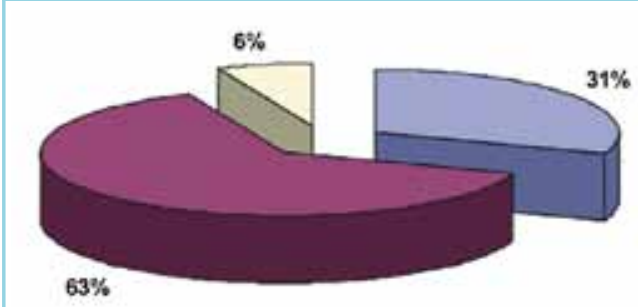

a05 to $07 \quad \mathbf{0} 03$ to $10 \quad a>11$ 


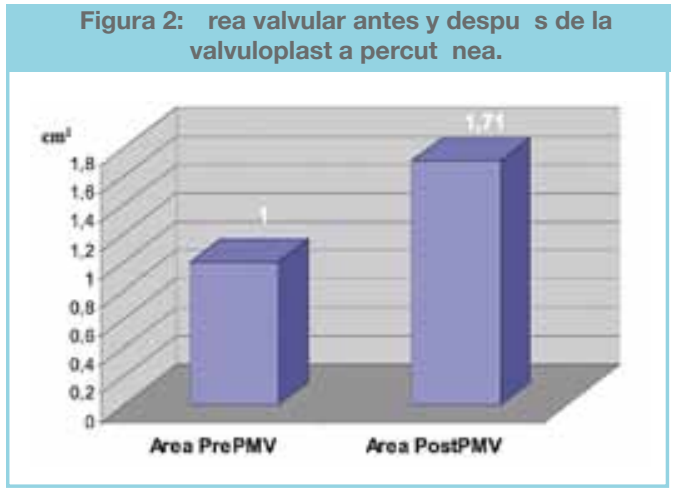

al taponamiento cardíaco. Once pacientes $(4,5 \%)$ desarrollaron insuficiencia mitral severa, ninguno de los cuales requiere cirugía inmediata. Diez de ellos fueron sometidos a reemplazo valvular mitral, uno de ellos rechazaron la cirugía y murió meses después.

Durante el periodo de seguimiento, 53 pacientes presentaron reestenosis $(21,6 \%)$, la que no mostró ninguna correlación con la edad, el resultado de la dilatación (optima versus sub-optima) o la presencia de fibrilación auricular. Solo la puntuación del Wilkins demostró ser predictivos de reestenosis (OR 1.28, IC 1,01-1,6 p $\leq$ $0,03)$ (tabla 1).

Antes de la dilatación, 131 pacientes $(53,5 \%)$ estaban en clase funcional (CF) I o II según la clasificación de New York Heart Association (NYHA). Al final del seguimiento, $220(89,8 \%)$ estaban en clase funcional I o II ( $\mathrm{p} \leq 0,001)$ (Figura 3).

Durante el período de observación, 5 pacientes fallecieron $(2,04 \%), 22(8,9 \%)$ necesitaron reemplazo de la válvula mitral y $11(4,5 \%)$ una segunda VMP debido a la reestenosis. La supervivencia libre de eventos en 3 años fue de $84,5 \%$. No se encontraron variables clínicas y ecocardiográficas para predecir estos eventos.

\section{Discusión:}

La VMP ofrece una alta tasa de éxito inicial con mejoría sintomática significativa, según lo reportado previamente por nuestro grupo ${ }^{8}$, así como por otros autores 22-24.

Estos resultados fueron confirmados en nuestra serie, $89 \%$ de los pacientes estaban en clase funcional I-II al final del seguimiento (Figura 3). La mortalidad asociada con el procedimiento fue baja $(1,02 \%)$, con sólo una muerte atribuida al mismo. Esta tasa de mortalidad es coherente con la literatura revisada 23,25 .

En nuestra serie, el desarrollo de la insuficiencia mi-
Tabla 1: Predictores de riesgo de reestenosis.

\begin{tabular}{lcccc} 
& OR & SE & $p$ & $\mathrm{Cl} 95 \%$ \\
\hline Dilataci n auricular & 0.50 & 0.21 & 0.10 & $0.22-1.14$ \\
Edad & 0.98 & 0.02 & 0.53 & $0.95-1.02$ \\
Fibrilaci n auricular & 1.55 & 0.61 & 0.27 & $0.71-3.37$ \\
Score de Wilkins & 1.28 & 0.15 & 0.03 & $1.01-1.6$ \\
\hline
\end{tabular}

OR:odds ratio. SE: Desviacion standard. p: p value. CI: Intervalo de confianza.

tral severa $(4,5 \%)$ post VMP fue ligeramente mayor que la reportado en otras series en Chile o América del Sur $(3,7 \%)^{1,28}$. La reestenosis detectada durante un seguimiento promedio de 3 años fue de $21,6 \%$, lo que concuerda con lo reportado por otros grupos - $40 \%$ en 6 años en la serie de Wang et al ${ }^{29}, 39 \%$ en 7 años en la serie de Hernández et al ${ }^{22}$, pero mayor que la de Fawzy et al, $19 \%$ en 7 años y $50 \%$ en 15 años ${ }^{25}$. En todas estas publicaciones, como en nuestra serie actual, la reestenosis tuvo una buena correlación con la puntuación de Wilkins, con un valor de $<8$ como predictivo de ausencia de reestenosis en el seguimiento tardío. A diferencia de otros, no encontramos ninguna correlación con la edad u otras variables clínicas ${ }^{5}$.

El 84,5\% de los pacientes estuvieron libre de eventos a los 3 años, lo que está en consonancia con recientes publicaciones $\left(92 \%\right.$ a los 5 y $87 \%$ a los 7 años ${ }^{25}$. La puntuación de Wilkins se ha utilizado para predecir la supervivencia libre de eventos en estudios a largo plazo 5,26,27. En esos estudios, la edad, características clínicas y la morfología de la válvula mitral se asociaron con los resultados, a diferencia de nuestra serie, donde sólo las características morfológicas fueron predictivos de la supervivencia libre de eventos a los 3 años.

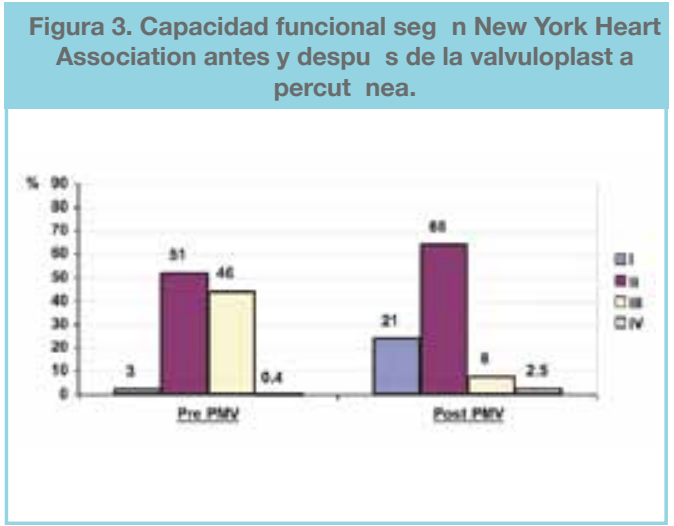




\section{Conclusiones:}

En la población de pacientes estudiados, la VMP tuvo una tasa aceptable de complicaciones atribuibles al pro- cedimiento. Las características ecocardiográficas de la válvula mitral se correlacionaron con la reestenosis. $\mathrm{La}$ incidencia de eventos tardíos fue baja, sin correlación con variables clínicas o morfológicas.

\section{Referencias:}

1. Garcia A, Treviño A, Ibarra M, Palacios J, Uribe A, De La Fuente $\mathrm{F}$, et al. Insuficiencia mitral post-valvuloplastia mitral con cateter balon: inicidencia, factores predictors y pronosticos. Arch. Inst. Cardiol. Mex 1995; 65: 39-47.

2. Olessen KH. The national history of 271 patients with mitral stenosis under medical treatment. Br Heart J 1962; 22: 349-352.

3. Rowe JC, Bland EF, Sprague HB, White PD. The course of mitral stenosis without surgery: ten- and twenty-year perspectives. Ann Intern Med 1960; 52: 741-749.

4. Roy SB, Gopinath N. Mitral stenosis. Circulation 1968; 38: 6876.

5. Palacios I. Farewell to surgical mitral commissurotomy for many patients. Circulation 1998; 97:223-226.

6. Ben Farhat M, Ayari M, Maatouk F, Betbout F, Gamra H, Jarra $\mathrm{M}$, et al. Percutaneous balloon versus surgical closed and open mitral commissurotomy. Seven-year follow-up results of a randomized trial. Circulation 1998; 97: 245-250.

7. Vahanian A, Michel PL, Cormier B, Vitoux B, Michel X, Slama M, et al. Results of percutaneous mitral commissurotomy in 200 patents. Am J Cardiol 1989; 63: 847-852.

8. Dighero H, Zepeda F, Sepúlveda P, Soto JR, Aranda W. Percutaneous Mitral Balloon Valvotomy: Six year Follow-up. J Invasive Cardiol 2001; 13: 795-799.

9. GorlinR, Gorlin S. Hydraulic formula for calculation of the area of the stenotic mitral valve, other cardiac valves and central circulatory shunts. Am Heart J 1951; 41: 1-29.

10. Hatle L, Angelsen BAJ, Tromsdal A. Noninvasive assessment of atrioventricular pressure half time by Doppler ultrasound. Circulation 1979; 1096-1104.

11. Wilkins GT, Weyman AE, Abascal VM, Block PC, Palacios IF. Percutaneous balloon dilatation of the mitral valve. An analysis of echocardiographic variables related to the outcome and the mechanism of dilation. Br Heart J 1988; 60: 299-308.
12. Sellers RD, Levy MJ, Amplatz K, Lillehei CW. Retrograde cardioangiography in acquired heart disease: indication and interpretation of 700 cases. Am J Cardiol 1964; 14: 437-447.

13. Inoue K, Owaki T, Nakamura T, Kitamura F, Miyamoto N. Clinical application of transvenous mitral commissurotomy by a new balloon catheter. J. Thorac Cardiovasc Surg 1984; 87: 394-402.

14. Al Zaibag M, Ribeiro PA, Al Kasab S, Al Fagih MR. Percutaneous double-balloon mitral valvotomy for rheumatic mitralvalve stenosis. Lancet 1986; 1: 757-761.

15. Abascal VM, Wilkins GT, O'Shea JP, Choong CY, Palacios IF, Thomas JD, et al. Prediction of successful outcome in $130 \mathrm{pa}-$ tients undergoing percutaneous mitral valvotomy. Circulation 1990; 82: 448-456.

16. Desideri A, Vanderperren O, Serra A, Barraud P, Petitclerc R, Lespérance J, et al. Long-term (9 to 33 months) echocardiographic follow-up after successful percutaneous mitral commissurotomy. Am J Cardiol 1992; 69: 1602-1606.

17. Dean LS, Mickel M, Bonan R, Holmes DR Jr, O’Neill WW, Palacios IF, et al. Four year follow-up of patients undergoing percutaneous balloon mitral commissurotomy. A report from the National Heart, Lung and Blood Institute Balloon Valvuloplasty Registry. J Am Coll Cardiol 1996; 28: 1452-1457.

18. Osa A, Almenar L, Rincón de Arellano A, Martí S, Roldán I, Mora $\mathrm{V}$, et al. Resultados a largo plazo de la valvuloplastía mitral percutánea. Rev Esp Cardiol 1998; 51: 458-466.

19. Olmos A, Seguel I, Gajardo J, Saldaña A, Lecannelier E, Beyer R, et al. Valvuloplastía mitral percutánea: Resultados inmediatos y tardíos en 300 pacientes. Rev Med Chile 1994; 122: 282-293.

20. Pan M, Medina A, Suárez de Lezo J, Hernández E, Romero M, Pavlovic D, et al. Factors determining late success after mitral balloon valvotomy. Am J Med. 1993; 71: 1181-1185.

21. Astudillo R, Pimienta G, Pech C. Valvuloplastía mitral percutánea con balón de Inoue. Resultados inmediatos y seguimiento a mediano plazo. Resúmenes de trabajos. II Congreso de la Sociedad Latinoamericana de Cardiología Intervencionista y VII Simposio Internacional del Colegio Argentino de Cardiólogos 
Intervensionistas. Agosto 1997. Revista de la Sociedad latinoamericana de Cardiología Intervensionista 1997; 2: 49.

22. Hernandez R, Bañuelos C,Alfonso F, Goicolea J, Fernández-Ortiz A, Escaned J, et al. Long-term clinical and echocardiographic follow-up after percutaneous mitral valvuloplasty with the Inoue balloon. Circulation. 1999; 99: 1580-1586.

23. Palacios IF, Sanchez PL, Harrell LC, Weyman AE, Block PC. Which patients benefit from percutaneous balloon valvuloplasty? Pre valvuloplasty and post valvuloplasty that predict long-term outcome. Circulation 2002; 105: 1465-1471.

24. Ben-Farhat M, Betbout F, Gamra H, Maatouk F, Ben-Hamda K, Abdellaoui M, et al. Predictors of long-term event-free survival freedom from restenosis after percutaneous balloon mitral commissurotomy. Am Heart J. 2001; 142: 1072-1079.

25. Fawzy ME, Hegazy H, Shoukri M, El Shaer F, ElDali A, AlAmri M. Lon-term clinical and echocardiographic results after successful mitral balloon valvotomy and predictors of long-term outcome. Eur Heart J. 2005; 26: 1647-1652.

26. Cohen DJ, Kuntz RE, Gordon SP, Piana RN, Safian RD, McKay RG, et al. Predictors of long-term outcome after percutaneous mitral valvuloplasty. N Eng J Med. 1992; 327: 1329-1335.

27. Lung B, Garbarz E, Michaud P, Helou S, Farah B, Berdah P, et al. Late results of percutaneous mitral commissurotomy in a series of 1024 patients: analysis of late clinical deterioration: frequency, anatomic findings and predictive factors. Circulation. 1999; 99: 3272-3278.

28. Martinez A, Gonzales A, Fajuri A, Marchant E, Guarda E, Pérez O, et al. Evolución alejada post valvuloplastía mitral percutanea: 10 años de seguimiento. Bol Escuela de Medicina, 2005; 30: 8-11.

29. Wang A, Krasuski RA, Warner JJ, Pieper K, Kisslo KB, Bashore TM, et al. Serial Echocardiographic Evaluation of Restenosis after Succesful Percutaneous Mitral Commissurotomy. J Am Coll Cardiol. 2002; 328-334. 